\title{
Immediate effect of different modes of one-session vibration on propriception of knee joint.
}

\author{
Qiu Yepeng ${ }^{*}$, Zhang Tingran ${ }^{2}$ \\ ${ }^{1}$ College of Physical Education, Chong Qing Normal University, PR China \\ ${ }^{2}$ Key Laboratory of Physical Evaluation and Sports Performance Monitoring of the State Administration of Sports, \\ College of Physical Education, Southwest University, PR China
}

\begin{abstract}
Purpose: To explore the influence of two vibration training methods (static and dynamic vibrations) on proprioception of knee-joint.

Method: Recruiting 12 healthy male students (similar in height, weight, and age) in Southwest University and adopting angular instrument and JVC camcorder to monitor knee-joint proprioception of subjects who have received different vibration trainings, so as to reveal vibration training's change to knee-joints' active and passive proprioception in open and closed-kinetic-chain modes.

Results: According to active and passive angle tests on open-kinematic-chain knee-joint and passive angle test on closed-kinetic-chain knee-joint of legs, error in non-intervention mode is remarkably larger than that in dynamic and static vibration intervention modes when the subjects are tested in these three modes. The comparison among the three intervention modes show that the dynamic vibration training is superior to vibration training whereas the static training is superior to no training according to the error in active and passive angle tests on open-kinematic-chain knee-joint and passive angle test on closed-kinetic-chain of dominant leg.

Conclusion: Vibration training exerts remarkable effect on improvement of proprioception of knee-joint and it is more suitable to adopt dynamic vibration training.
\end{abstract}

Keywords: One-session vibration, Knee joint, Sport.

Accepted on July 10, 2017

\section{Introduction}

It is the common knowledge that the formation of athletic skills is a complicated nervous process in four stages i.e. movement generalization, differentiation, consolidation, and automation. When the movement enters into automation stage, the athlete can complete complex movements by virtue of precise muscular sense instead of vision. However, the athlete's precise muscular sense forms on the basis of proprioception. In addition, it is demonstrated by a great many domestic and overseas researches that proprioception training methods can be employed in injury prevention and rehabilitation of athletes. Also, it's proved that proprioception can be improved through training, which in turn will improve the stability of nervemuscle reflex system and improve sport performance and avoid sport injury. As an emerging strength training method, vibration training garners increasing attention from experts at home and abroad for it can effectively enhance muscular strength and explosive force with relatively small load. According to a range of researches, Bosco et al. [1] found that vibration training cannot only help improve the muscle's maximum strength but also has positive effects on the muscle's rapid strength and strength endurance. Then some scholars held that $[2,3]$ vibration stimulation can change hormone level in men's blood and thus exert positive influence on improvement of the subjects' balance ability, leaping ability, and proprioception. Trans' research finds that [4] vibration training is a time-saving, safe, and high-efficiency method to improve muscular strength and proprioception for those women suffering from gonitis. Zeng [5] has conducted researches on knee-joint's proprioception characteristics in open and closed-kinetic-chain modes but failed to find the influence of different vibration training methods on kneejoint's proprioception. This paper aims to make efforts in this regard and thus provide significant references for enriching of vibration training practice and enhancement of subjects' proprioception.

\section{Subjects and Method}

\section{Subjects}

12 right-footed male students from Southwest University are recruited for the test. And the subjects should have no organic disease and no medical history in knee-joint and lower limb injury, and receive no professional and vibration trainings of 
any kind. Prior to the test, we will inform the subjects of the whole test process and the notice items, and sign test agreements with them after their approval. During the test, these volunteers should keep normal eating and living habits.

\section{Method}

Test procedure: This study is aimed at making a discussion on vibration training's instant effect on knee-joint's proprioception and self-control of single sample is adopted in the experimental design, so there should be a time interval of 3 weeks after each test method, in order to eliminate the influence from previous training. All subjects will receive 3 tests ( $6 \mathrm{~d}$ in total).

Test I: All subjects receiving no training will receive tests of three modes $n$ a quiet and relaxed state. And the test data will be recorded.

Test II: A time internal is unnecessary since there is no vibration in Test I. Test II can be conducted after $2 \mathrm{~d}$ of Test I. Prior to the test, the subjects are required to do warm up at their original positions and then they will receive static vibration training. After the test, relevant parameters will be measured.

Test III: After test II, the subjects are required to have a rest of $5 \mathrm{~d}$, so as to eliminate the influences from vibration effects of Test II. Similarly, the subjects are required to warm up prior to the test and then they will receive dynamic vibration training. After the test, relevant parameters will be measured instantly.

\section{Vibration mode specification}

1) Dynamic vibration training: Power plate (manufactured in Germany) will be employed in the test (whole-body vibration method; vibration frequency: $40 \mathrm{HZ}$; amplitude: $4 \mathrm{~mm}$; vibration time of each time: $3 \mathrm{~min}$; time interval between groups: 3 min; 6 times). In each vibration training, the subjects are required to jump up and down for 1 min between vibration platform and ground along the front and rear direction and for 2 min between vibration platform and ground along the left and right direction.

2) Static vibration training intervention: The vibration training instrument used in dynamic training will be employed in the test (whole-body vibration method; vibration frequency: $40 \mathrm{HZ}$; amplitude: $4 \mathrm{~mm}$; vibration time: $30 \mathrm{~s}$; time interval between groups: $1 \mathrm{~min}$; 6 times). In the vibrating training, the subjects are required to stand on the vibration platform with half-squat posture, during which they should stand on their tiptoes with both hands surrounded by the chest.

\section{Data acquisition method}

Two domestically-manufactured electronic angular instruments will be respectively put at the position which is at $1 / 3$ (far end) of the outer thigh's central line and $1 / 3$ (near end) of the outer shank's central line. The included angle between the central lines of the two electronic angular instruments is the angle of knee-joint. Herein, the electronic angular instrument can record the angle change of the knee-joint at each moment.

\section{Result}

\section{Proprioception test of dominant leg in different intervention methods}

After the subject's non-intervention, dynamic vibration training intervention, and static vibration training intervention, absolute value of error between dominant leg's active angle test and preintervention test in knee-joint's open-kinetic-chain mode is respectively $4.38 \pm 1.76^{\circ}, 3.03 \pm 1.23^{\circ}$ and $3.25 \pm 1.64^{\circ}$. Error of non-intervention is remarkably larger than that of dynamic vibration training intervention and static vibration training intervention. After the subject's non-intervention, dynamic vibration training intervention, and static vibration training intervention, absolute value of error between dominant leg's passive angle test and pre-intervention test in knee-joint's open-kinetic-chain mode is respectively $3.97 \pm 2.11^{\circ}, 2.06 \pm$ $1.12^{\circ}$ and $32.64 \pm 1.57^{\circ}$. Error of non-intervention is remarkably larger than that of dynamic vibration training intervention and static vibration training intervention. And error of dynamic vibration training intervention is remarkably smaller than that of static vibration training intervention.

\section{Test result of non-dominant leg's proprioception in different interventions}

After the subject's non-intervention, dynamic vibration training intervention, and static vibration training intervention, absolute value of error between non-dominant leg's active angle test and pre-intervention test in knee-joint's open-kinetic-chain mode is respectively $6.42 \pm 1.76^{\circ}, 3.52 \pm 2.01^{\circ}$ and $3.12 \pm 1.52^{\circ}$. Error of non-intervention is remarkably larger than that of dynamic vibration training intervention and static vibration training intervention. After the subject's non-intervention, dynamic vibration training intervention, and static vibration training intervention, absolute value of error between non-dominant leg's passive angle test and pre-intervention test in knee-joint's open-kinetic-chain mode is respectively $5.67 \pm 2.11^{\circ}, 3.13 \pm$ $1.34^{\circ}$ and $2.96 \pm 1.36^{\circ}$. Error of non-intervention is remarkably larger than that of dynamic vibration training intervention and static vibration training intervention. And error of dynamic vibration training intervention is remarkably smaller than that of static vibration training intervention.

\section{Discussion}

In vibration-free, dynamic vibration and static vibration, active and passive angle test on dominant leg's open-kinematic-chain knee-joint and passive angle test on closed-kinematic-chain knee-joint show that error of non-intervention is remarkably larger than that of dynamic and static vibration intervention, indicating that vibration training can significantly improve knee-joint's proprioception. This phenomenon is similar to the result of test which was conducted by Topp who adopted a method similar to passive angle test on closed-kinematic-chain knee-joint. According to Topp's test, proprioception of kneejoint in vibration training group was remarkably better than that in non-vibration group, but there was no difference 
between vibration team and non-vibration team in active angle test on open-kinematic-chain knee-joint, which was different from the current test result. That can probably be attributed to the test object. In Topp's research, the test object is menopausal women while this research focuses on young men who outperform menopausal women in athletic ability, nervous excitation, and muscle adaptability. Roelants conducted vibration training on 17 tennis players and found that vibration training cannot only help improve proprioception of ankle joint but also remarkably enhance [6] accuracy of ankle joint inversion and valgus. According to Huang's [7] $8 \mathrm{w}$ vibration training with different frequencies on basketball players, the subjects' knee-joint topesthesia is better than that of control group. Altman held that [8] vibration training is mainly aimed at weakening vibration and maintaining body balance and thus strengthening the training to nerve-muscle system, which has activated kinematic units that have not been recruited before. In this way, strength and proprioception are improved. However, there are some scholars who got the opposite result that vibration training decreases the athletes' proprioception instead of improving it [9].

According to the results arising from different vibration methods, this research finds that dynamic vibration is more effective than static vibration in improving the proprioception of knee-joint. The test shows that result of dynamic vibration training is superior to that of static vibration training in both active and passive angle test on dominant leg's openkinematic-chain knee-joint and passive angle test on closedkinematic-chain knee-joint, which is consistent with Roelants research result. Currently, most domestic scholars hold that dynamic vibration training is more effective than static vibration training in improving balance ability, coordinating ability, muscular endurance, and nerve excitability, etc., which has been proved by current research. However, there are also contrary results; for example, according to the research of Wolfe, the influence of dynamic vibration training and static vibration training on the body's proprioception and balance ability is basically the same. According to the view in this paper, the difference of scholars' research results can be mainly attributed to different vibration parameters. For this reason, it's necessary to standardize these parameters in future research, so as to further make definite the different effects between dynamic vibration and static vibration.

\section{Conclusion}

Be it dynamic or static vibration, both active and passive angle test on dominant leg's open-kinematic-chain knee-joint and passive angle test on closed-kinematic-chain knee-joint are all smaller than non-vibration in error. According to the comparison between the effect of dynamic vibration training and that of static vibration training, dynamic vibration is superior to static vibration training. In active open-kinematic- chain mode, passive closed-kinematic-chain mode, and active closed-kinematic-chain mode, proprioception is different in error i.e. error of active angle in closed-kinematic-chain mode is minimum, whereas error of passive angle in pen-kinematicchain mode is maximum.

\section{References}

1. Bosco JS, Greenleaf JE, Bernauer EM, Card DH. Effects of acute dehydration and starvation on muscular strength and endurance. Acta Physiol Pol 1974; 25: 411-421.

2. Bosco C, Iacovelli M, Tsarpela O, Cardinale M, Bonifazi M, Tihanyi J, Viru M, De Lorenzo A, Viru A. Hormonal responses to whole-body vibration in men. Eur $\mathrm{J}$ Appl Physiol 2000; 449-454.

3. Riccardo Di Giminiani, Leila Fabiani, Giuliano Baldini, Giovanni Cardelli, Aldo Giovannelli, Jozsef Tihanyi. Hormonal and Neuromuscular Responses to Mechanical Vibration Applied to Upper Extremity Muscles. Plos one 2014.

4. Segal NA, Glass NA, Shakoor N, Wallace R. Vibration platform training in women at risk for symptomatic knee osteoarthritis. J Injury Function Rehab 2013.

5. Zeng C, li H, Yang T, Deng ZH, Yang Y, Zhang Y, Lei GH. Electrical stimulation for pain relief in knee osteoarthritis: systematic review and network meta-analysis. Osteoarthritis Cartilage 2015; 23: 189-202.

6. Brown K, Loprinzi PD, Brosky JA, Topp R. Prehabilitation influences exercise-related psychological constructs such as self-efficacy and outcome expectations to exercise. J Strength Condition Res 2014; 28: 201-209.

7. Xiaoguang Z. Effects of increasing physical activity on foot structure and ankle muscle strength in adults with obesity. J Physical Ther Sci 2016; 28: 2332-2336.

8. Wang HH, Chen WH, Liu C, Yang WW, Huang MY, Shiang TY. Whole-body vibration combined with extraload training for enhancing the strength and speed of track and field athletes. J Strength Cond Res 2014; 28: 2470-2477.

9. Pozo-Cruz BD, Adsuar JC, Parraca JA, Pozo-Cruz JD, Olivares PR, Gusi N. Using whole-body vibration training in patients affected with common neurological diseases: a systematic literature review. J Alter Complement Med 2012; 18: 29-41.

\section{*Correspondence to}

Qiu Yepeng

College of Physical Education

Chong Qing Normal University

PR China 